\title{
An Optimization Method for Train Flow Adjustment between Parallel Routes on Railway Network
}

\author{
Boliang Lin ${ }^{1, a}$, Jianping Wü ${ }^{1, b}$, Siqi Liu ${ }^{1, c}$,Jiaxi Wang ${ }^{1, d}$, Chang Liu ${ }^{1, \text { e }}$ \\ ${ }^{1}$ School of Traffic and Transportation, Beijing Jiaotong University, Beijing100044, China. \\ abllin@bjtu.edu.cn, b15114205@bjtu.edu.cn, '15114206@bjtu.edu.cn, \\ d13114236@bjtu.edu.cn, e14114241@bjtu.edu.cn
}

Keywords: train flow adjustment, damping coefficient adjustment method, train bypass scheme.

\begin{abstract}
This paper focuses on the train flow adjustment between parallel routes on railway network. We firstly analyze the classical arc-path models based on the multi-commodity flow. According to the analysis of the mathematical model, we propose a practical method, namely the damping coefficient adjustment method (DCAM). We extend the concept of the shortest route from narrow sense to broad sense by setting damping coefficients, then we use the shortest path method to research the train flow adjustment between parallel routes. Furthermore, this paper presents some methods and principles for setting damping coefficients. We finally provide an example to prove the reasonableness and effectiveness of DCAM.
\end{abstract}

\section{Introduction}

The long-term pressure will damage the integrity and stability of railroad track, thus leading to overhaul or mid-level maintenance. In addition, emergent accidents and natural disasters may block a partof railway lines and sometimeseven all the network. Both of the overallmaintenance and emergencies may block the operation of rolling stocks and decrease the carrying capacity of related segments. In this case, a portion of trains which are previously designed to pass through the affected lines have to make a detour or stay in station. If parallel routes of the blocked lines exist, the issue of adjusting train flow between parallel routes will be important and of great research significance no matter in theory or practice.

From the perspective of optimization, train route adjustment issue can be solved by arc-path model proposed for multi-commodity flow. As the adjustment has a high requirement on locality and real-time performance, it is a direct-viewing and practical method to bring damping coefficient into decision making when addressingthe train flow adjustment issue resulted from natural disasters or emergencies.

\section{Analysis of Arc-path Model for Train Route Optimization}

There are $\mathrm{N}$ stations in a railway network. And $b_{m}$ denotes the $m$-arc's capacity which is represented by the number of trains passing through the arc in a whole day. $M$-arc's generalized cost is denoted by $c_{m}$. Let $f_{i j}$ denotes the number of trains from station $i$ to station $j$. To ensure the generality of our model we assume that the size of all trainsis a constant. And we define $l_{i j}$ as the set of possible pathwayscontaining the shortest path, the second-shortest path and so on. For the $k$ route, the operation cost can be expressed by

$c_{i j}^{k}=\sum a_{i j}^{m k} c_{m}(1)$

In the formula, the arc-path associated variable $a_{i j}^{m k}$ can be defined as

$a_{i j}^{m k}=\left\{\begin{array}{lc}1 & m \text {-arc on the } k \text {-route from } i \text { to } j_{(2)} \\ 0 & \text { or }\end{array}\right.$

We define decision variables of train path as follows:

$x_{i j}^{k}=\left\{\begin{array}{lc}1 & \text { train from } i \text { to } j \text { choose the } k \text {-route } \\ 0 & \text { or }\end{array}\right.$

The objective function and constraint conditions can be expressed as follows: 


$$
\begin{aligned}
& \min Z=\sum_{i} \sum_{j} \sum_{k} f_{i j} c_{i j}^{k} x_{i j}^{k} \\
& \sum_{\text {s.t. }}^{k} x_{i j}^{k}=1 \quad \forall i, j \\
& \sum_{i}^{k} \sum_{j} \sum_{k} f_{i j} a_{i j}^{m k} x_{i j}^{k} \leq b_{m} \quad \forall m \\
& x_{i j}^{k} \in\{0,1\} \quad \forall i, j, k
\end{aligned}
$$

The objective of formula (4) is to minimize the sum of generalized costs for train operation. The constraint (5) ensures that only one route in set $l_{i j}$ can be chosen for a certain train. The constraint (6) guarantees that the number of trains through the arcwould not exceed its capacity.

Butthis model involvesa plenty of variables and it will take lots of time forcomputation. And sometimes, the outcome of this model is not consistent with the empirical judgments of transportation engineering. Thus, the problem is not going to be solvedby the approach above mentioned. According to the analysis of the mathematical model, a convenient and practical method called damping coefficient adjustment method will be introduced.

\section{The Damping Coefficient Adjustment Method (DCAM)}

The basic principle of DCAM is assigning different values to the damping coefficients of different routes to change the unit transport cost of each path, thereby, adjusting the travel routes of trains in railway network. The damping coefficient increases (or decreases) the generalized mileage of the related pathway in practice. The formula is given as follows:

$$
L_{\tau}=\tau L
$$

where $L_{\tau}$ is the generalized mileage; $L$ is the actual distance and $\tau$ denotesthe damping coefficient.

As shown in Fig. 1, we need to get the practical distribution information of train flows at the first step, and assign it to the network so that we can initialize the network. Then, we adjust the routes of trainsaccording to requirements and judge whether the set still remains adjustment-needed train or not. If there is no train needing adjustment, we should output the adjustment scheme.Otherwise we have to go to the next step. In this step, we reconstruct the network by removing the saturated arcs. Then, we should judge whether there are parallel routes in network. If not, we have to stop and output the adjustment scheme.Otherwise we set the damping coefficient according to relative methods and principles which will be analyzed in detailin the next section. Then, we sort the route in descending order by generalized mileage and select the new route for adjustment-needed trains. Thus, we achieve an effective circulation of adjustment process until a satisfied adjustment scheme is obtained. Flow chart of DCAM is as follows: 


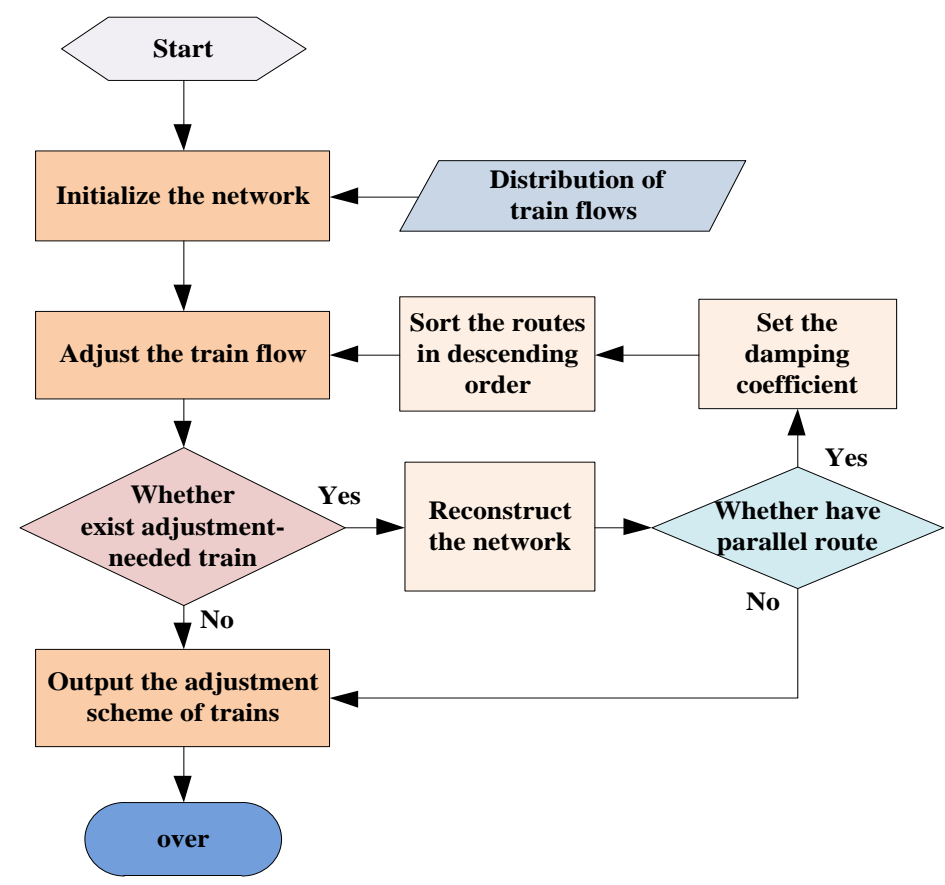

Fig. 1 Flow chart of DCAM

\section{Methods and Principles for Setting Damping Coefficient}

The key of damping coefficient adjustment method lies in the determination of damping coefficient of related paths. Various factors influence the determination of damping coefficient.In this part, we will study the determination of damping coefficient in detail.

Train Flows with the Restraint of Arrival Deadline and Short Distance Are Inclined to ChoosePaths with Low Detour Rate.Firstly,we should introduce a new conception, namely detour rate. Detour rate is the ratio of $\mathrm{D}$-value (determined by the mileage of the alternative path and the shortest path) to the mileage of the shortest path. $l_{0}$ is the mileage of the shortest path for a certain transport task; $l_{1}$ is the mileage of an available route for detour. The equation is as follows:

$=\left(l_{1}\right.$

$\left.-l_{0}\right)$

$/ l_{0} \times 100 \%$

It can be concluded that the smaller the value (outcome of this formula) is, the shorter the detour mileage will be.

In modern logistics systems, customers are strict with the arrival deadline of commodities. How to ensure the timely arrival of commodities when facing occasional detour is an inevitable problem for train flow dispatchers. To address this problem, we could assign a relatively small value to the damping coefficient of each available alternative which ensures the timely arrival of commodities. Thus, these available paths have a priority in adjustment (more likely to be chosen as detour route). Furthermore, the mileage of path can also influence the value assigned to each damping coefficient. The receptivity of detour rate of trains for long distance service is stronger than the trains for short distance service. That is to say, short distance trains are more likely to choose the parallel paths with small $\eta$ value, which has to be taken into consideration.

Railway Lines with Larger Tonnage Rating Have Priority in Adjustment. In the adjustment, the available alternatives with the same or larger tonnage rating than the original path enjoy a priority in adjustment. Trains do not need to be detached when operating in this kind of paths and can directly pass through. However, when the tonnage rating of the chosen alternative is smaller than the original one, trains have to be detached, which will certainly increase the time of transportation. In conclusion, when setting damping coefficients, we should assign bigger values to the available paths with equivalent or larger tonnage rating, while smaller value to the path with 
smaller tonnage rating. Moreover, we should comprehensively analyze the operation time of trains in the path without detachment and path with detachment, and assign relatively smaller value to the damping coefficient of the less time-consuming path. In this way, we can obtain reasonable damping coefficients of various routes.

Cargo First and Passenger Later. During holidays and particularly the Spring Festival, China Railways Corporation will temporarily run additional trains to satisfy passengers' travelling demand, whichreduces the carrying capacity of some segments of railway paths designed for passengers and cargos. In this case, we should take the influence of different kinds of trains into consideration when setting damping coefficients for available alternative paths. On the one hand, we could increase the value of damping coefficients of passenger trains and give them a priority to pass through. On the other hand, we can set different damping coefficients according to the levels of passenger trains and ensure the passing priority of high-level passenger trains.

\section{Case Study}

In this section, we construct a simple railway network containing parallel paths which is shown in Fig.2. It can be concluded that this network consist of 9 stations and 12 running sections. There are three parallel paths from station $A$ to station $F$, namely path $1(A \rightarrow B \rightarrow C \rightarrow D \rightarrow E \rightarrow F)$, path 2 $(\mathrm{A} \rightarrow \mathrm{B} \rightarrow \mathrm{G} \rightarrow \mathrm{E} \rightarrow \mathrm{F})$ and path $3(\mathrm{~A} \rightarrow \mathrm{H} \rightarrow \mathrm{I} \rightarrow \mathrm{F})$

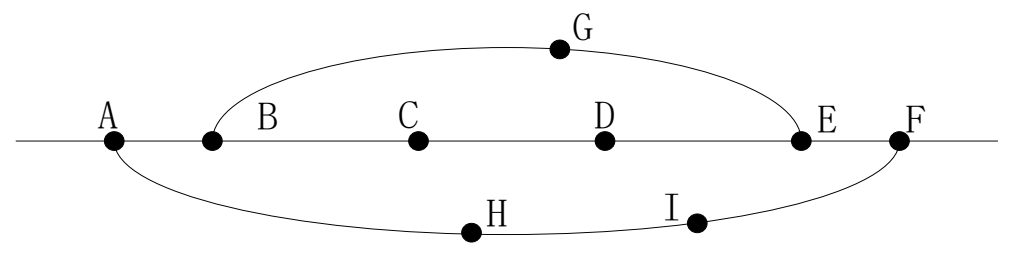

Fig. 2 A simple railway network

As shown in Fig.2, the carrying capacity of path 1 is 30 trains. Because of the special geological condition and delayed maintenance in section $\mathrm{C} \rightarrow \mathrm{D}$, the devices and equipments are seriously worn out and need necessary maintenance which is expected to last a month. In this case, the carrying capacity of $\mathrm{C} \rightarrow \mathrm{D}$ section has reduced 20\%, which means 6 trains have to make a detour through other paths or be out of service. At present, two parallel paths of path 1 have surpluscarrying capacities. Path 2 can pass through 5 more trains and path 3 has potential for accepting 4 more trains. So, we adjust the excessive 6 trains to path 1 and path 2 . We assume that the levels of these 6 trains are the same. It will take at least 4 hours to detach trains at station B and the detailed data of attributes of all the parallel paths are shown in table 1.

Table 1 Detailed data of all the parallel paths

\begin{tabular}{ccc}
\hline Path & Tonnage rating/t & Operating mileage/km \\
\hline 1 & 5000 & 425 \\
2 & 4500 & 470 \\
3 & 5000 & 520 \\
\hline
\end{tabular}

According to the principles of setting damping coefficient, we set the value of damping coefficients of path 1 , path 2 and path 3 as 100,1.2 and 1.0 respectively. To facilitate the programming of computer, we set the maximum value of damping coefficient as 100 . Based on the damping coefficients acquired just now, we could calculate the generalized mileage of the three paths, which is $52500 \mathrm{~km}, 564 \mathrm{~km}$ and $520 \mathrm{~km}$ respectively. Given that the carrying capability surplus of path 3 is 4 trains, we could adjust 4 trains from path 1 to path 3 . As there are trains still in the need of adjustment, it is a must to set damping coefficient again for the related paths according to the principles above mentioned. We know that the damping coefficients of path 1 and path 2 are 100 and 1.2, respectively and the generalized mileages of these two paths are $42500 \mathrm{~km}$ and $564 \mathrm{~km}$. Now, the number of trains that need adjustment is 2 and the carrying capability surplus of path 2 is 4 trains, which can fully meet the demand of path 1 . After the latest adjustment, there are no trains that need adjustment. So the final plan is: transfer 4 freight trains of path 1 to path 3 and transfer the 
other two freight trains to path 2.

\section{Literature References}

Main research works, both domestically and internationally, are as follows: Paper [1, 2] studied the set of reasonable paths of railway network and proposed the arc-path model for the attribution of optimal train flow path. Paper [3] took the comprehensive appraisal of adjustment plan as its study object and carried out an in-depth research on the basis of scheduling command with a systematic viewpoint. Paper [4] took the concept of multiple commodity flow into consideration and studied the scheduling optimization problem by constructing a virtual arc equation. Relative documents for train flow optimization have not been found yet and in most cases, railway dispatchers rely on their experience.

\section{Summary}

This paper analyzes the optimization problem of large scale traffic flow adjustment on complicated railway network. Firstly, this paper points out the deficiencies of a mathematical model and proposes theDCAMinspired by relative programming models. After application test, we find that the method proposed in this paper is really convenient in addressing the large scale train flow adjustment problem in China railway network and the outcome is satisfactory. However, we can't confirm the specific traffic flow in adjustmentyet, and the method needs further improvement.

\section{Acknowledgements}

This work was supported by the science and technology department of the China Railway Corporationunder Grant Number,2015X004-C, the Chinese Natural Science Foundation under Grant Number,51178031and the Fundamental Research Funds for the Central Universitiesunder Grant Number, 2015YJS075. I'd like to present my sincere gratitude to Ms. Wen Shao from Beijing Normal University for her advice on the refinements and embellishments of English writing.Boliang Lin isthe corresponding author, and the communication email is bllin@bjtu.edu.cn.

\section{References}

[1] Lin, B.L., S.N. Song and Z.S. Chen. The 0-1 Programming Model for Traffic Flow Optimization and Algorithm for Generating Reasonable Route Set. [J]. Journal of Railway, 1997, 1 9(1):7-12

[2] Lin, B.L., H. Peng and B.G. Ren. Weighted Route Optimization Method for Traffic Flow in Railway Network. [J]. Journal of Northern Jiaotong University, 1996, 20(6):645-650

[3] Zhou,Y., Q.Y. Peng. Research of Comprehensive Appraisal and Relative Problems in Railway Adjustment. [D]. 2010

[4] Tian, Y.M., B.L. Lin and L.J. Ji. Research of Node-Arc Model and Arc-Path Model for Railway Traffic Flow Adjustment Based on Multiple Commodity Flow and Virtual Arc. [J] Journal of Railway, 2010, 3(4) 